\title{
BIOINDICADORES: UTILIZAÇÃO DE MACRÓFITAS AQUÁTICAS PARA AVALIAÇÃO DE AMBIENTES LACUSTRES
}

Francine Manrique Canhizares dos Santos, Welliton Leandro de Oliveira Boina

${ }^{1}$ Universidade do Oeste Paulista - UNOESTE, Curso de Pós-Graduação Latu Senso em Análise Ambiental Laboratorial e Biotecnologia, Presidente Prudente, SP.e-mail: weboina@gmail.com

\section{RESUMO}

O aumento excessivo da população e consequentemente o aumento desenfreado pelo uso dos recursos naturais, desencadeia uma série de impactos ao meio ambiente e esses impactos decorrentes das atividades humanas nos ecossistemas aquáticos vêm produzindo uma contínua e inexorável deterioração das águas. Um desses impactos é o processo de eutrofização, onde ocorre o aumento excessivo da toxicidade e o aumento na concentração de nutrientes, especialmente fósforo e nitrogênio. Este estudo objetivou apresentar as macrófitas aquáticas como uma importante ferramenta bioindicadora para a avaliação de ambientes lacustres, utilizando duas represas no Município de Martinópolis, SP. A metodologia constou de fotografias e avaliação dos ambientes lacustres em campo para posterior catalogação e comparação com ambientes ideais. A identificação das espécies seguiu a proposta de classificação das famílias reconhecidas pelo sistema de classificação The Angiosperm Phylogeny Group APG III (2009). Os resultados demonstram que o uso múltiplo dos reservatórios está comprometido. Assim, conclui-se que a implantação de estudos visando à indicação de macrófitas aquáticas como bioindicadoras da qualidade da água é prioritária e altamente recomendável.

Palavras-chave: ambiente lacustre, bioincadores, espécie, macrófita, eutrofização.

\section{BIOINDICATORS: USE OF AQUATIC MACROPHYTES FOR EVALUATION OF LACUSTRINE ENVIRONMENTS}

\begin{abstract}
Excessive population growth and hence the rampant increase the use of natural resources, triggers a series of environmental impacts and these impacts from human activities on aquatic ecosystems have produced a continuous and relentless deterioration of water. One such impact is the process of eutrophication, which occurs excessive increased toxicity and increased concentration of nutrients, especially phosphorus and nitrogen. This study aimed to present aquatic macrophytes as an important bio-indicator tool for the evaluation of lacustrine environments using a dam in the municipality of Martinópolis, SP. The methodology consisted of photographs and lacustrine environment evaluation in the field for later cataloging and comparison with ideal environments. Species identification followed the proposed classification of families recognized by the classification system The Angiosperm Phylogeny Group - APG III (2009). The results demonstrate that multiple use of the reservoirs is engaged. Thus, it is concluded that the implementation of studies for the indication of macrophytes as bioindicators of water quality is a priority and highly recommended.
\end{abstract}

Keywords: environment lake, biodindicators, species, macrophyte, eutrophication.

\section{INTRODUÇÃO}

As macrófitas aquáticas são originalmente vegetais que durante sua história evolutiva, sofreram modificações, onde retornaram do ambiente terrestre para 0 aquático, sendo capazes de colonizar lagos, lagoas, rios, represas, cachoeiras, zonas úmidas, etc ${ }^{1}$.

A vegetação encontrada nos ecossistemas aquáticos continentais é classificada em diversos grupos ecológicos: (i) emersas, (ii) flutuantes, (iii) enraizadas, (iv) submersas livres e (v) enraizadas com folhas flutuantes. Por esta vez, 
as macrófitas aquáticas desempenham importante função na manutenção e equilíbrio dos ambientes aquáticos, na produção da biomassa, no controle da erosão hídrica, no melhoramento físico e nutricional do solo e na ciclagem de nutrientes, também sendo uma ótima bioindicadora da qualidade da água, onde sua presença pode melhorar a qualidade das águas devido a sua capacidade de absorver cargas excessivas de nutrientes e outros elementos ${ }^{2}$.

Com o aumento das atividades humanas e o aumento desenfreado dos recursos naturais e a criação de represas artificiais, o aumento excessivo de nutrientes nos ambientes aquáticos é muito comum, se trata de um processo denominado eutrofização, com impacto de considerável efeito que ocorre o aumento da toxicidade e concentração dos nutrientes, especialmente fósforo e nitrogênio, podendo assim comprometer os usos múltiplos do ambiente aquático. A eutrofização pode ser natural ou artificial (cultural ou antrópica). Os efeitos negativos ocasionados pela eutrofização são vários, onde podemos destacar: 0 desenvolvimento intenso e descontrolado do fitoplâncton; degradação da qualidade da água com alterações de composição, cor, turbidez, transparência, aumento da decomposição orgânica, causando, consequentemente um consumo maior de oxigênio dissolvido até anoxia; liberação de gases e produção de maus odores; produção de substâncias tóxicas e prejuízos consideráveis para o uso em abastecimento, lazer, irrigação, turismo e paisagismo, entre outros ${ }^{3}$.

Levando as macrófitas aquáticas em consideração como bioindicadoras de qualidade, apresentam inúmeras vantagens quando comparado a outros índices de monitoramento da qualidade da água, tendo uma avaliação ambiental tanto mais segura quanto mais confiável, onde isso acontece porque as mesmas possuem aplicações práticas e são alternativas mais baratas e eficazes comparadas às outras análises físicas e químicas, que são mais sofisticadas com custos mais elevados ${ }^{4}$.

Com isso, o objetivo deste trabalho foi analisar se nos locais de estudo seriam encontradas espécies comumente consideradas indicadoras de ambiente eutrofizados, ou seja, um ambiente com carga excessiva de nutrientes e quais possíveis causas estariam contribuindo para o aumento desta característica no ambiente.

\section{METODOLOGIA}

O local utilizado para estudo foram duas represas artificiais, identificada como Represa A e Represa B, com áreas aproximadas de $561 \mathrm{~m}^{2}$ e $279 \mathrm{~m}^{2}$ respectivamente. Ambas as represas localizadas no Município de Martinópolis-SP, pertencentes a Bacia Hidrográfica do Rio do Peixe, situada dentro da Unidade de Gerenciamento de Recursos Hídricos - UGRHI 21. Foram feitas avaliações do ambiente lacustre em campo e registrado via fotografias digitais as espécies de macrófitas aquáticas mais predominantes encontradas no local de estudo. A identificação das espécies seguiu a proposta de classificação das famílias reconhecidas pelo sistema de classificação Angiosperm Phylogeny Group - APG III ${ }^{5}$. Atualmente é o mais moderno sistema para classificação das angiospermas, segundo critérios filogenéticos.

Posteriormente foi efetuado o processo de catalogação das espécies e comparação das mesmas em relação a ambientes lacustres ideais utilizando-se de levantamento bibliográfico especializado para as devidas avaliações.

\section{RESULTADOS}

Através da avaliação do ambiente lacustre, observação e registro fotográfico das plantas aquáticas foi possível classificar e identificar as espécies de macrófitas aquáticas nos dois locais (Represa A e Represa B) de estudo utilizando o sistema de classificação APG III $(2009)^{1,5}$. Contudo, foi considerado para classificação e identificação as plantas aquáticas mais predominantes por comparação via observação in loco. 


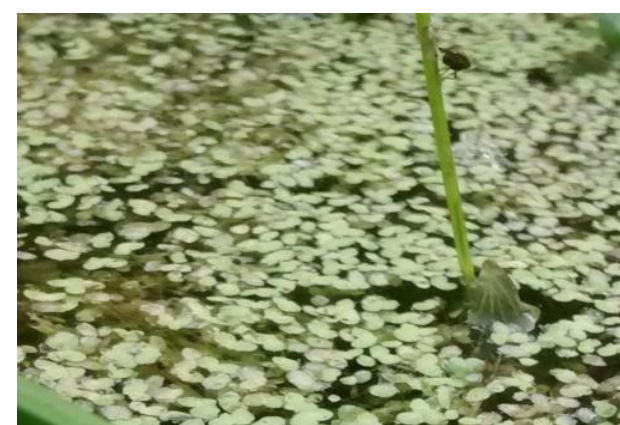

(a)

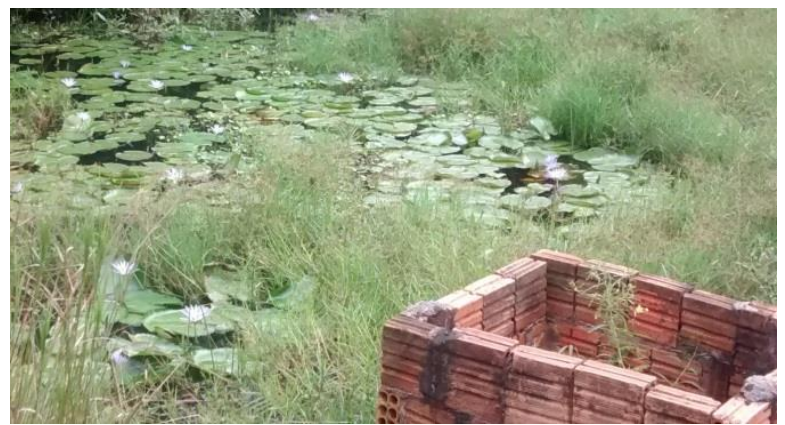

(b)

Figura 1. (a) Lemna valdiviana e (b) Nymphaea pulchella.

Conforme observações in loco na Represa A, foram identificadas duas espécies predominantes de macrófitas, a Lemna valdiviana apresentada na Figura 1a e a Nymphaea pulchella apresentada na Figura 1b. A espécie Lemna valdiviana, conhecida popularmente como lentilha-d'água, pertence à família Lemnaceae enquanto a espécie Nymphaea pulchella é pertencente à família Nymphaeaceae, ambas espécies possuem forma biológica flutuante na superfície.

Como pode ser observado na Figura 1, a superfície do ambiente aquático apresenta pouquíssimas áreas de lâmina d'água sem a presença de macrófitas.

Represa B:

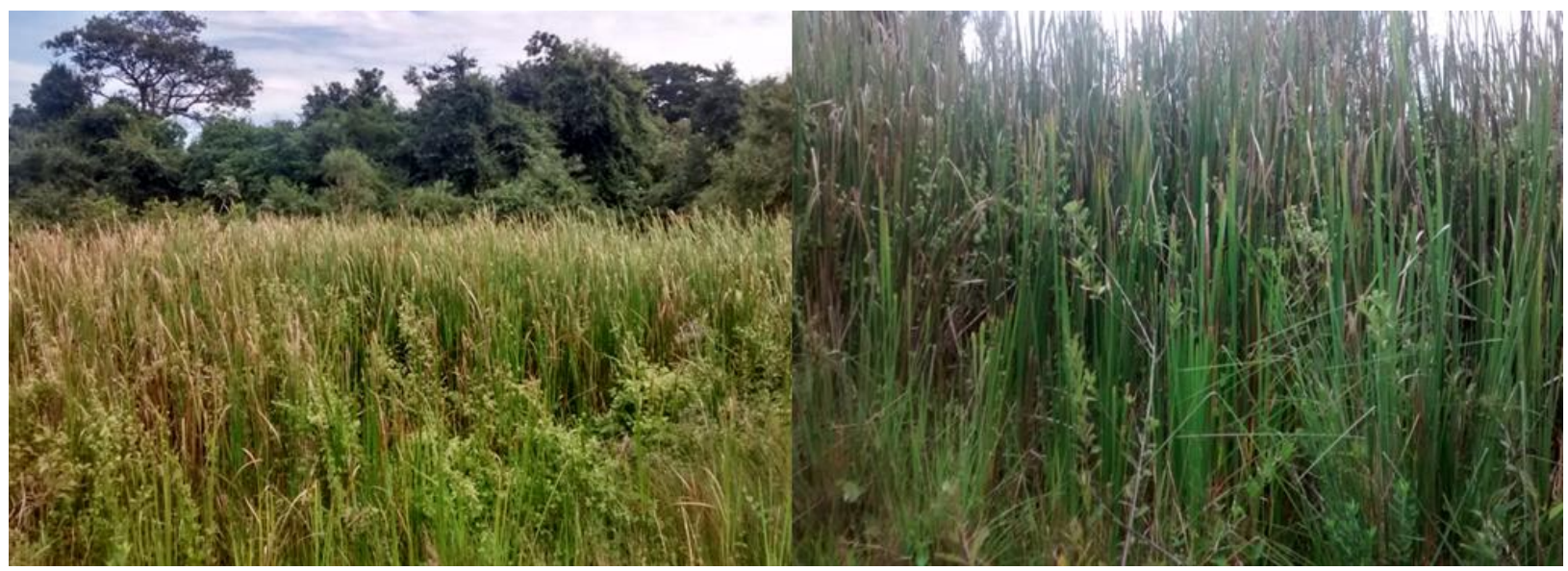

Figura 2. Typha domingensis.

Conforme observações in loco na Represa $B$, foi identificado uma espécie predominante de macrófita, a espécie Typha domingensis, conhecida popularmente por taboa, pertencente à família Typhaceae. Esta espécie possui forma biológica enraizada no sedimento, e de acordo com Silva ${ }^{6}$, apresenta uma série de característica, tais como tolerância a ambientes impactados, crescimento vegetativo rápido e alta produção de biomassa. Como pode ser observado na Figura 2, a superfície do ambiente aquático não apresenta áreas de lâmina d'água sem a presença de macrófita.

Cabe ressaltar, segundo Esteves', as macrófitas aquáticas são classificadas conforme duas formas de vida, tais como: emersas; flutuante fixa; flutuante livre; submersa fixa e submersa livre.

Entretanto, a distribuição e a abundância de macrófitas aquáticas são determinadas, entre outros fatores, pela composição dos sedimentos, turbidez da água, disponibilidade de nutrientes e ação de herbívoros ${ }^{7}$. Dentre esses fatores, ocorrem ainda, pressões antrópicas que podem induzir o aparecimento de condições favoráveis para o desenvolvimento desses vegetais, como a construção de reservatórios artificiais e a eutrofização.

\section{DISCUSSÃO}

As áreas estudadas, são ambientes artificiais, criados inicialmente para a criação de 
peixe, atualmente as áreas não são mais usadas para essa atividade. Podemos observar que, em volta das represas existe uma mata ciliar, que contribui também para o aumento de nutrientes na água devido a decomposição das folhas, aumentando assim a carga de nitrogênio e fósforo no ambiente aquático. Geralmente nos ambientes lênticos, as macrófitas aquáticas encontram condições favoráveis para o desenvolvimento o ano todo, devido possuir altas concentrações de nutrientes que favorece $o$ crescimento do fitoplancton e de macrófitas aquáticas flutuantes, como o caso da espécie Lemna sp encontrada na superfície da Represa A, que impede a penetração de luz na coluna d'água. Habitualmente, esses vegetais da família Lemnaceae vivem flutuando livremente sobre a superfície de lagoas ricas em matéria orgânica, sendo dispersas por aves aquáticas e correntezas ${ }^{8}$. São exigentes em relação a nutrientes, principalmente fontes de nitrogênio e fósforo, porém, se desenvolvidas em condições ideais, apresentam maior taxa de crescimento entre os vegetais superiores ${ }^{1,8}$.

A ocorrência da espécie Nymphaea pulchella, também na Represa $A$, são macrófitas emergentes, se tratam de vegetais enraizados que possuem folhas e flores flutuantes e que também formam grandes bancos de macrófitas sombreando o meio, impedindo o desenvolvimento de outros vegetais. Na Represa B, a predominância da macrófita Typha domingensis ocorre pelas características favoráveis do meio, onde a falta de herbívoros, a velocidade de corrente que permite sua estabilidade no solo, temperatura, disponibilidade de luz e a eutrofização, contribuem para o seu crescimento e propagação. São macrófitas aquáticas muito estudadas em áreas alagadas em relação a absorção de nutrientes, sendo fenotipicamente plástica e bem adaptada a ambientes eutróficos apresentando uma maior absorção de fósforo em ambientes impactados ${ }^{9}$.

O acréscimo excessivo dessas espécies, e de indivíduos de uma população de macrófitas deve-se geralmente, a dois fatores principais: à falta de herbívoros e ao nível de eutrofização'. Podemos notar nos ambientes estudados que ambos os fatores ocorrem, e a eutrofização no ambiente é a de processo natural, que está diretamente ligado no aumento progressivo e lento da sedimentação e na concentração de nitrogênio, fósforo e da matéria orgânica, onde esses estão relacionados com efeitos do vento, erosão por chuvas e adição de material biológico.

Contudo, esse aumento de nutrientes inferioriza a qualidade da água, comprometendo os usos múltiplos do ambiente aquático. Todavia, as espécies registradas nos ambientes são indicadoras de ambientes eutrofizados, onde a análise sugere $\mathrm{o}$ uso das macrófitas como parâmetro para o monitoramento da qualidade da água, visto que a utilização de macrófitas aquáticas como bioindicadoras pode ser realizado pelo indicativo da presença ou ausência, como também pela análise de parâmetros, tais como tamanho da população ou comunidade, forma e atributos funcionais ${ }^{10}$.

\section{CONCLUSÃO}

As espécies de macrófitas aquáticas consideradas bioindicadoras de ambientes eutrofizados estão presentes nos locais de estudo (Lemna valdiviana, Nymphaea pulchella e Typha domingensis) e os fatores como temperatura, luz e disponibilidade de nutrientes contribuem para o desenvolvimento dessas macrófitas, onde seu aumento excessivo acarreta a eutrofização do ambiente lacustre.

Consequentemente, uma vez que qualidade da água é afetada, o uso múltiplo dos reservatórios estará comprometido. Assim, o uso dessas macrófitas como bioindicadoras em ambientes lacustres é de extrema importância para o monitoramento da qualidade da água, entretanto deve-se fazer o controle efetivo das mesmas com a finalidade de prevenir seu crescimento excessivo e evitar o processo de eutrofização para que a qualidade da água possa ser recuperada.

\section{CONFLITOS DE INTERESSE}

Os autores declaram não haver qualquer potencial de conflito de interesse que possa interferir na imparcialidade deste trabalho científico.

\section{REFERÊNCIAS}

1. Esteves FA. Fundamentos de Limnologia. 2.ed. Rio de Janeiro: Interciência; 1998.

2. Santino MBC, Bianchini Junior I. Colonização de macrófitas aquáticas em ambientes lênticos [Monografia]. São Carlos: Universidade Federal de São Carlos - UFSCar, 2011.

3. Pompeo M. Monitoramento e manejo de plantas aquáticas. Oecol. Bras. 2008;12(3):40624. 
4. Silva SSL. Caracterização ecológica e estrutural de macrófitas em reservatórios no estado de Pernambuco [Tese]. Pernambuco: Universidade Federal Rural de Pernambuco - UFRPE, 2011.

5. Angiosperm Phylogeny Group (2009). An update of the Angiosperm Phylogeny Group classification for the orders and families of flowering plants: APG III. Botan J Linnean Soc. 2009;161(2):105-21.

DOI:

https://doi.org/10.1111/j.1095-

\subsubsection{6.x}

6. Silva CO. Características anatômicas e fisiológicas de Typha domingensis Pers. Submetida a concentrações crescentes de arsênio. [Dissertação]. Lavras: Universidade Federal de Lavras, 2014.

7. Junior IB. Modelos de crescimento e decomposição de macrófitas aquáticas. In: Thomaz SM, Bini LM (Eds.). Ecologia e manejo de macrófitas aquáticas. Maringá: EDUEM; 2003. p.86-126.

8. Mohedano RA. Tratamento de efluente e produção de alimento, em cultivos de tilápias (Oreochromis niloticus), através da macrófita aquática Lemna valdiviana (lemnaceae). - Uma contribuição para a sustentabilidade da aquicultura [Dissertação]. Florianópolis: Universidade Federal de Santa Catarina, 2004.

9. Rocha CMC, Alves AF, Cardoso AS, Cunha MCC. Macrófitas aquáticas como parâmetro no monitoramento ambiental da qualidade da água. Rev Bras Geog Fís. 2012;5(4):970-83.

10. Pedralli G. Macrófitas aquáticas como bioindicadoras da qualidade da água: alternativas para usos múltiplos de reservatórios. In: Thomaz SM, Bini LM (Eds.). Ecologia e manejo de macrófitas aquáticas. Maringá: EDUEM; 2003. p.171-88.

Recebido para publicação em 16/08/2016

Revisado em 29/08/2016

Aceito em 08/09/2016 